\title{
Insulin \& C-Peptide Levels in Sulfonylurea-Induced Hypoglycemia: A Systematic Review
}

\author{
Christopher R. DeWitt, MD ${ }^{a, b, c}$, Kennon Heard, MD ${ }^{d, e, f, g}$, Javier C. Waksman, $M D^{h}$
}

\author{
aSaint Paul's Hospital, Division of Emergency Medicine, Vancouver, BC, Canada \\ bBritish Columbia Poison Information Centre, Vancouver, BC, Canada \\ cUniversity of British Columbia, Vancouver, BC, Canada \\ dUniversity of Colorado Division of Emergency Medicine, Denver, CO \\ eUniversity of Colorado School of Medicine, Denver, CO \\ fThe Rocky Mountain Poison and Drug Center, Denver, CO \\ gColorado Emergency Medicine Research Center, Denver, CO \\ hUniversity of Colorado School of Medicine and Newfields, Denver, CO
}

\section{ABSTRACT}

Objectives: We will describe insulin and C-peptide levels observed in sulfonylurea-induced hypoglycemia and determine whether these levels differed if obtained before or after hypoglycemic therapy.

Methods: We performed a systematic review of the English literature to identify Medline articles containing "sets" (glucose $<60 \mathrm{mg} / \mathrm{dL}$ with insulin and C-peptide levels). These "sets" were categorized as being obtained BEFORE, AFTER, or UNKNOWN with respect to hypoglycemic therapy.

Results: 22 articles, 76 patients, and 97 "sets" were included. Mean glucose (mg/dL), insulin ( $\mu \mathrm{IU} / \mathrm{mL})$, and C-peptide (ng/mL) for all "sets' were 28.6 ( $\pm 12.6 ; 26.1$ to 31.2$), 54.4$ ( $\pm 126.3 ; 28.3$ to 80.5$), 7.2$ ( $\pm 6.2 ; 5.9$ to 8.5 ).

- The BEFORE measures were 24.3 ( $\pm 7.3 ; 18.7$ to 30.0 ), 36.6 ( $\pm 26.2 ; 16.5$ to 56.7 ), 5.4 ( $\pm 4.6 ; 1.5$ to 9.2).

The AFTER measures were 33.1 ( $\pm 9.8 ; 28.2$ to 38.0), 126.7 ( $\pm 278.1 ; 0$ to 265.0$), 10.3$ ( $\pm 10.5 ; 5.1$ to 15.4 ).

- The UNKNOWN measures were 28.0 ( $\pm 13.5 ; 24.7$ to 31.3$), 37.1$ ( $\pm 21.8 ; 31.7$ to 42.5$), 6.5$ ( $\pm 4.3 ; 5.4$ to 7.6 ).

Only one "set" (glucose $49 \mathrm{mg} / \mathrm{dL}$ ) had insulin $<3.9 \mu \mathrm{IU} / \mathrm{mL}$ and C-peptide $<1.4 \mathrm{ng} / \mathrm{mL}$.

Conclusions: Insulin $\geq 3.9 \mu \mathrm{IU} / \mathrm{mL}$, C-peptide $\geq 1.4 \mathrm{ng} / \mathrm{mL}$, and glucose $<49 \mathrm{mg} / \mathrm{dl}$ are consistent with sulfonylurea-induced hypoglycemia. BEFORE levels were lower, but they were consistent with sulfonylurea-induced hypoglycemia.

\section{INTRODUCTION}

Measurement of insulin and C-peptide levels may be employed in the investigation of an unknown cause of hypoglycemia. When insulin is secreted, equimolar amounts of insulin and

C-peptide (the peptide fragment cleaved from proinsulin) are released into circulation [1]. Increased endogenous insulin secretion is associated with increased C-peptide secretion, whereas insulin levels are high and C-peptide levels low with exogenous insulin administration $[2,1]$. Thus, serum insulin and C-peptide
\end{abstract}

Keywords: sulfonylurea, hypoglycemia, insulin, C-peptide

Acknowledgements: We would like to thank Dr. PY Kwong for kindly supplying previously unpublished data used in our study. Dr. Kwong had no financial interest in this study.

Notes: There was no outside funding of any kind used for this study.

Corresponding Author: Christopher R. DeWitt, Saint Paul's Hospital Division of Emergency Medicine. Email: cdewittmd@yahoo.com 
measurements may be exploited to differentiate between endogenous (insulinoma or medications that stimulate insulin secretion) and exogenous (insulin administration) insulin sources. Because sulfonylurea medications stimulate the secretion of insulin, and necessarily C-peptide, sulfonylurea-induced hypoglycemia may mimic hypoglycemia caused by an insulinoma [2-6].

Many medical texts and published algorithms tell us sulfonylurea-induced hypoglycemia yields insulin and C-peptide levels that are "high" or "elevated," but the texts and published algorithms fail to provide details; such as the magnitude of the effect and the effect of hypoglycemic treatment on expected values [3,7-9] Other authors provide suggested cut-off values for insulin and C-peptide without reference to values actually observed $[4-6,10,11]$. The primary purpose of this study was to describe the range of observed values for these variables in sulfonylureainduced hypoglycemia and determine whether these variables differed if levels were obtained before or after hypoglycemic therapy was administered. We accomplished this aim by performing a systematic review of the English literature to identify cases that report measured serum glucose, insulin, and C-peptide levels associated with sulfonylurea-induced hypoglycemia.

\section{METHODS}

We performed asystematic review of the English literature from 1970 (C-peptide assay first described) through March 2004 [12]. The Ovid search engine (Ovid Technologies ${ }^{\mathrm{TM}}, 2000-2003$ ) was used to search the Medline database. Our Medical Subject Headings (MeSH) included the following: "hypoglycemia" AND "sulfonylurea compounds" OR "C-peptide" OR "insulin," OR the text words "hypoglycemi\$" AND "sulfonylurea\$," OR "C-peptide," OR "insulin" for human exposures. A broad search was used because articles identified before the search was initiated had variable MeSH headings. But nearly all were categorized under "hypoglycemia" and/or "sulfonylurea compounds," "C-peptide", or "insulin." The search criteria identified eleven thousand forty-two articles.

The primary author reviewed the titles and/or abstracts of all articles retrieved by the search. We included articles if hypoglycemia was due to sulfonylureas (confirmed by history or laboratory analysis) and if insulin and C-peptide levels were obtained at the time of documented hypoglycemia (blood glucose of $<60 \mathrm{mg} / \mathrm{dL}$ (3.3 mmol/L) via bedside glucometer or venous sampling). We excluded articles if cases involved another hypoglycemic agent in addition to a sulfonylurea, as well as patients with hepatic or renal failure because insulin is mainly cleared by the liver and C-peptide by the kidney $[9,13,14]$. The primary author reviewed, in full, the articles that were unable to be included or excluded based on title or abstract.

All authors reviewed articles that met the inclusion criteria to confirm inclusion criteria and to classify patients into groups; (1) those who had "sets" obtained BEFORE hypoglycemic therapy, or (2) those who had "sets" AFTER hypoglycemic therapy. A "set" was defined as blood glucose ( $<60 \mathrm{mg} / \mathrm{dL} ; 3.3 \mathrm{mmol} / \mathrm{L})$, insulin, and C-peptide levels that were obtained at the same time.
Hypoglycemic therapy included administration of oral or intravenous glucose, glucagon, octreotide, or diazoxide. If it was unknown or unclear when hypoglycemic therapy was administered compared to when "sets" were obtained, they were included in a third UNKNOWN group. The algorithm for article acquisition is shown in Figure 1.

Levels reported in SI units were converted to conventional units using the following conversions; glucose: $\mathrm{mmol} / \mathrm{L}=0.0555$ $\mathrm{mg} / \mathrm{dL}$; insulin: $\mathrm{pmol} / \mathrm{L}=6.945 \mu \mathrm{IU} / \mathrm{mL} ; \mathrm{nmol} / \mathrm{L}=0.333 \mathrm{ng} / \mathrm{mL}$.

Statistical Analysis: All analyses were done using GraphPad Instat version 3.06 (@ 1992-2003 GraphPad Software, Inc.). For all articles meeting inclusion criteria, we performed descriptive statistics using all patients and all "sets" of levels. Additionally, descriptive statistics were performed on each group, (BEFORE therapy, AFTER therapy, and UNKNOWN). However, the articles did not provide the ages and sex of many patients. Additionally, several glucose, insulin, and C-peptide levels were reported as non-exact levels (such as values reported as $<$ or $>$ ). These levels are not included in mean or median calculations, but they are included in range descriptions.

\section{RESULTS}

Through the Ovid search, we identified 19 articles that met the inclusion criteria. Reference lists of these 19 articles were handsearched, and 4 additional articles meeting inclusion criteria were found: this yielded 23 articles. One article by Hussain et al. was excluded by group consensus because we were unable to determine which blood glucose measurements corresponded to reported insulin and C-peptide levels [16]. This yielded a total of 22 articles. The articles provided cases of 76 patients with 97 "sets" of levels that met inclusion criteria. All authors agreed on the classification of each case into the BEFORE, AFTER or UNKNOWN groups. Information for all included articles and cases is shown in Table 1.

A single C-peptide value (in the BEFORE therapy group) was reported as $12,100 \mathrm{ng} / \mathrm{mL}$ [20]. This level was 270 times higher than the next highest level, and considered erroneous by the authors. It was excluded from all calculations, but it is shown in Table 1 for completeness. Finally, the article by Hampton et al. reported only mean glucose, insulin, and C-peptide levels of 3 patients. These levels are not included in descriptive statistics, but they are included in Table 1.

Table 2 shows the descriptive statistics of all articles that met inclusion criteria, and Table 3 shows the statistics of the BEFORE, AFTER, and UNKNOWN groups. There is a wide variation in reported insulin and C-peptide levels in sulfonylurea-induced hypoglycemia. Although glucose, insulin, and C-peptide levels tended to be higher in the AFTER therapy group, there was wide overlap with results in the BEFORE therapy group.

\section{DISCUSSION}

Although the medical literature tells us insulin and C-peptide levels are elevated in sulfonylurea-induced hypoglycemia, there is a 


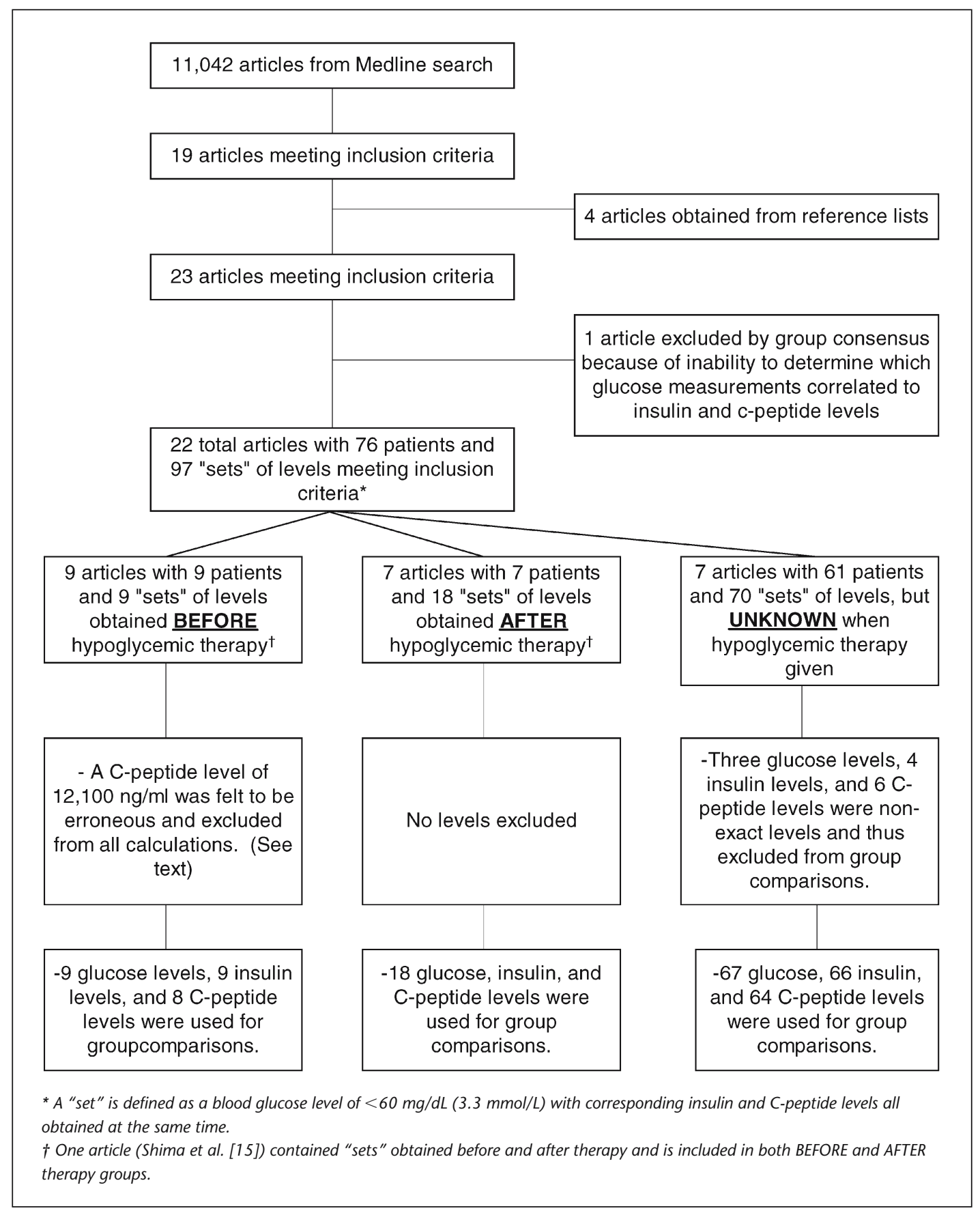

Figure 1: Algorithm for article acquisition.

lack of information regarding levels that are actually observed $[3,7,8]$. However, we must first understand what actually constitutes an "elevated" insulin or C-peptide level in a hypoglycemic setting. It is not that levels are above normal fasting ranges, but they are relatively greater than what would be expected at a given blood glucose level. Normal fasting ranges do not apply to hypoglycemic patients $[10,11]$. As blood glucose levels fall around $45-60 \mathrm{mg} / \mathrm{dL}$, insulin and C-peptide secretion nearly ceases $[3,17,38]$. Failure to suppress insulin and C-peptide secretion, in the setting of hypoglycemia, indicates inappropriate endogenous insulin secretion.

We must also realize that laboratories utilize different assay techniques. Older polyclonal insulin assays cross-react with proinsulin and insulin precursors, yielding insulin levels higher than the newer monoclonal assays $[4,9,14]$. However, there is still a large amount of variability in the sensitivity and cross-reactivity of monoclonal assays, and universally accepted levels for the 
Table 1: Articles meeting inclusion criteria

\begin{tabular}{|c|c|c|c|c|c|c|c|}
\hline Article & Age/sex & $\begin{array}{l}\text { Glucose } \\
(\mathbf{m g} / \mathbf{d L})\end{array}$ & $\begin{array}{l}\text { Insulin } \\
(\mathbf{m l U} / \mathbf{m L})\end{array}$ & $\begin{array}{l}\text { C-peptide } \\
(\mathbf{n g} / \mathbf{m L})\end{array}$ & $\begin{array}{l}\text { Assay for } \\
\text { Insulin and } \\
\text { C-peptide }\end{array}$ & $\begin{array}{l}\text { Sulfonylurea } \\
\text { involved }\end{array}$ & Circumstances \\
\hline Cryer[17] & $64 / M$ & 36 & 15 & 1.5 & Not provided & Glipizide & $\begin{array}{l}\text { Accidentally dispensed } \\
\text { instead of nifedipine }\end{array}$ \\
\hline Krentz[18] & $36 / \mathrm{M}$ & 18 & 41 & 4.6 & "Double-antibody RIAs" & Tolbutamide & $\begin{array}{l}\text { Intentional ingestion in } \\
\text { non-diabetic }\end{array}$ \\
\hline Labib[19] & $90 / F$ & 13 & 3.9 & 2.5 & $\begin{array}{l}\text { "competitive } \\
\text { double antibody } \\
\text { radio-immuno-assay" }\end{array}$ & Glyburide & $\begin{array}{l}\text { Presumably inadvertent } \\
\text { administration by } \\
\text { husband }\end{array}$ \\
\hline Ludman[20] & $65 / F$ & 20 & 85.8 & $12100 \dagger$ & Not provided & Chlorpropamide & $\begin{array}{l}\text { Accidentally dispensed } \\
\text { instead of acetazolamide }\end{array}$ \\
\hline Manning[21] & $47 / F$ & 34 & 15.1 & 3.2 & Not provided & Glyburide & $\begin{array}{l}\text { Presumably intentional } \\
\text { administration by } \\
\text { husband }\end{array}$ \\
\hline Sener[23] & $23 / \mathrm{M}$ & 23 & 52.1 & 6.3 & Not provided & Glipizide & $\begin{array}{l}\text { Presumably inadvertent } \\
\text { administration by } \\
\text { girlfriend }\end{array}$ \\
\hline Shima[15] & $23 / F$ & 25 & 25 & 3.5 & $\begin{array}{l}\text { "plasma } \\
\text { immuno-reactive } \\
\text { insulin (IRI) and } \\
\text { C-peptide immuno- } \\
\text { reactivity (CPR)" }\end{array}$ & Tolbutamide & Not identified \\
\hline
\end{tabular}

Comments: Levels were drawn during a $100 \mathrm{~g}$ oral glucose tolerance test after an overnight fast. The first "set" of levels was obtained at the same time glucose was given, and thus considered BEFORE therapy. Subsequent levels were obtained every 30 minutes thereafter and are included in the AFTER therapy group.

\begin{tabular}{lllllll}
\hline Sledge[24] & $72 / \mathrm{M}$ & 27 & 30 & 5.4 & Not provided & $\begin{array}{l}\text { Acetohexamide } \\
\text { Accidentally dispensed } \\
\text { instead of acetazolamide }\end{array}$ \\
\hline
\end{tabular}

Articles with levels drawn AFTER hypoglycemic therapy administered

$\begin{array}{ccccl}\text { Christesen[25] Newborn/ } 23 & 49 & 6.2 & \begin{array}{l}\text { "DELFIA analysis } \\ \text { (two-site two-step }\end{array} & \begin{array}{l}\text { Tolbutamide } \\ \text { M }\end{array} \\ & & & \begin{array}{l}\text { time resolved immuno- } \\ \text { fluorometric assay)" }\end{array} & \begin{array}{l}\text { tolbutamide for } \\ \text { gestational diabetes } \\ \text { when patient was born }\end{array}\end{array}$

Comments: Born with hypoglycemia. Levels drawn 3 hours after birth while receiving glucose and octreotide infusions. Three additional complete "sets" were drawn, but excluded because glucose $\geq 60 \mathrm{mg} / \mathrm{dL}$. Five incomplete "sets" excluded (no insulin level obtained).

\begin{tabular}{lllllll}
\hline Earle[26] & $82 / \mathrm{F}$ & 33 & 72 & 44.1 & Not provided & $\begin{array}{l}\text { Glimepiride } \\
\text { administered by } \\
\text { husband }\end{array}$
\end{tabular}

Comments: $10 \%$ dextrose infusion was held for "a short time." The patient then became symptomatic and these levels were drawn.

\begin{tabular}{llllllll}
\hline Goudie[27] & $56 / \mathrm{M}$ & 52 & 50 & 11.4 & Not provided & Glyburide & $\begin{array}{c}\text { Adulterant in herbal } \\
\text { medication }\end{array}$
\end{tabular}

Comments: Received $1 \mathrm{mg}$ IM glucagon 1 hour before levels drawn, and was eating "sugary" foods when levels drawn.

\begin{tabular}{|c|c|c|c|c|c|c|c|}
\hline Shenfield[28] & $85 / F$ & 43 & 14.6 & 5.4 & Not provided & Chlorpropamide & $\begin{array}{l}\text { Thought due to a } \\
\text { dispensing error }\end{array}$ \\
\hline
\end{tabular}

Comments: Receiving "continuous dextrose infusion" when levels drawn.

\begin{tabular}{llllllll}
\hline Teale[29] & 40/F & 32 & 17.9 & 3.8 & Not provided & Glyburide
\end{tabular}

Comments: Receiving "continuous intravenous glucose infusion" when levels drawn. 
Table 1: (Continued)

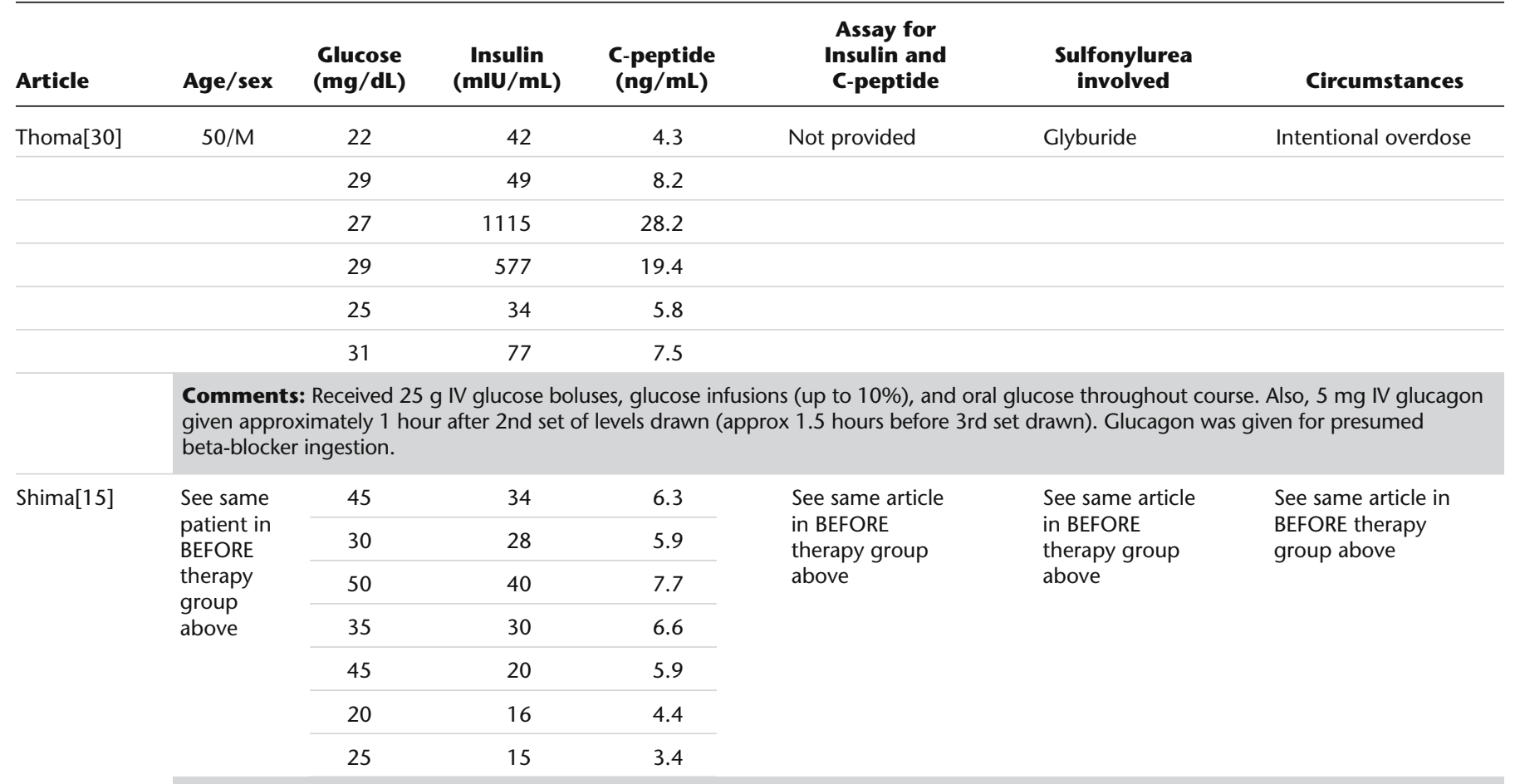

Comments: These levels were drawn at 30 minute intervals after oral glucose administered.

Articles where it is UNKNOWN when levels drawn with respect to hypoglycemic therapy

\begin{tabular}{|c|c|c|c|c|c|c|c|}
\hline Ahlquist[31] & $70 / F$ & 40 & 102 & 10.2 & Not provided & Tolazamide & $\begin{array}{l}\text { Accidentally dispensed } \\
\text { instead of tolmetin }\end{array}$ \\
\hline & \multicolumn{7}{|c|}{ Comments: May have been receiving glucose infusion when levels drawn, but unclear. } \\
\hline
\end{tabular}

Comments: Unknown if patients received therapy before levels drawn. Exact levels for each patient are unknown.

\begin{tabular}{|c|c|c|c|c|c|c|c|}
\hline Klonoff[33] & $38 / \mathrm{F}$ & 43 & 39.4 & 6.6 & Not provided & Glyburide & $\begin{array}{l}\text { Presumably confused } \\
\text { with friends' } \\
\text { furosemide tablets }\end{array}$ \\
\hline
\end{tabular}

\begin{tabular}{|c|c|c|c|c|c|c|c|}
\hline Kwong [34] & $\begin{array}{l}\text { Exact ages } \\
\text { unknown } \\
\text { but ranged } \\
\text { from }>21 \text { to } \\
>91 \text { yrs. Sex } \\
\text { unknown } \\
\text { for all } \\
\text { patients. }\end{array}$ & 13 & 26.2 & 5.7 & $\begin{array}{l}\text { "Insulin was } \\
\text { measured by } \\
\text { an established } \\
\text { in-house double- } \\
\text { antibody } \\
\text { competitive } \\
\text { RIA ..." }\end{array}$ & $\begin{array}{l}\text { Glyburide in } \\
7 \text { patients }\end{array}$ & $\begin{array}{l}\text { Ten cases of suspected } \\
\text { or proven factitious } \\
\text { drug ingestion: } 1 \text { with } \\
\text { intentional ingestion, } \\
1 \text { 'accidentally' took } \\
\text { wife's medication, } \\
2 \text { with Munchausen's, } \\
6 \text { others suspected, } \\
\text { but not proven. }\end{array}$ \\
\hline
\end{tabular}

Comments: Levels are not included in article, but were provided by author. (Kwong PY, personal communication March 23, 2004)

$\begin{array}{lll}27 & 2.2 & \\ & & \text { "C-peptide was } \\ & \text { measured by a } \\ & \text { competitive } \\ & \text { RIA kit..." }\end{array}$




\section{Table 1: (Continued)}

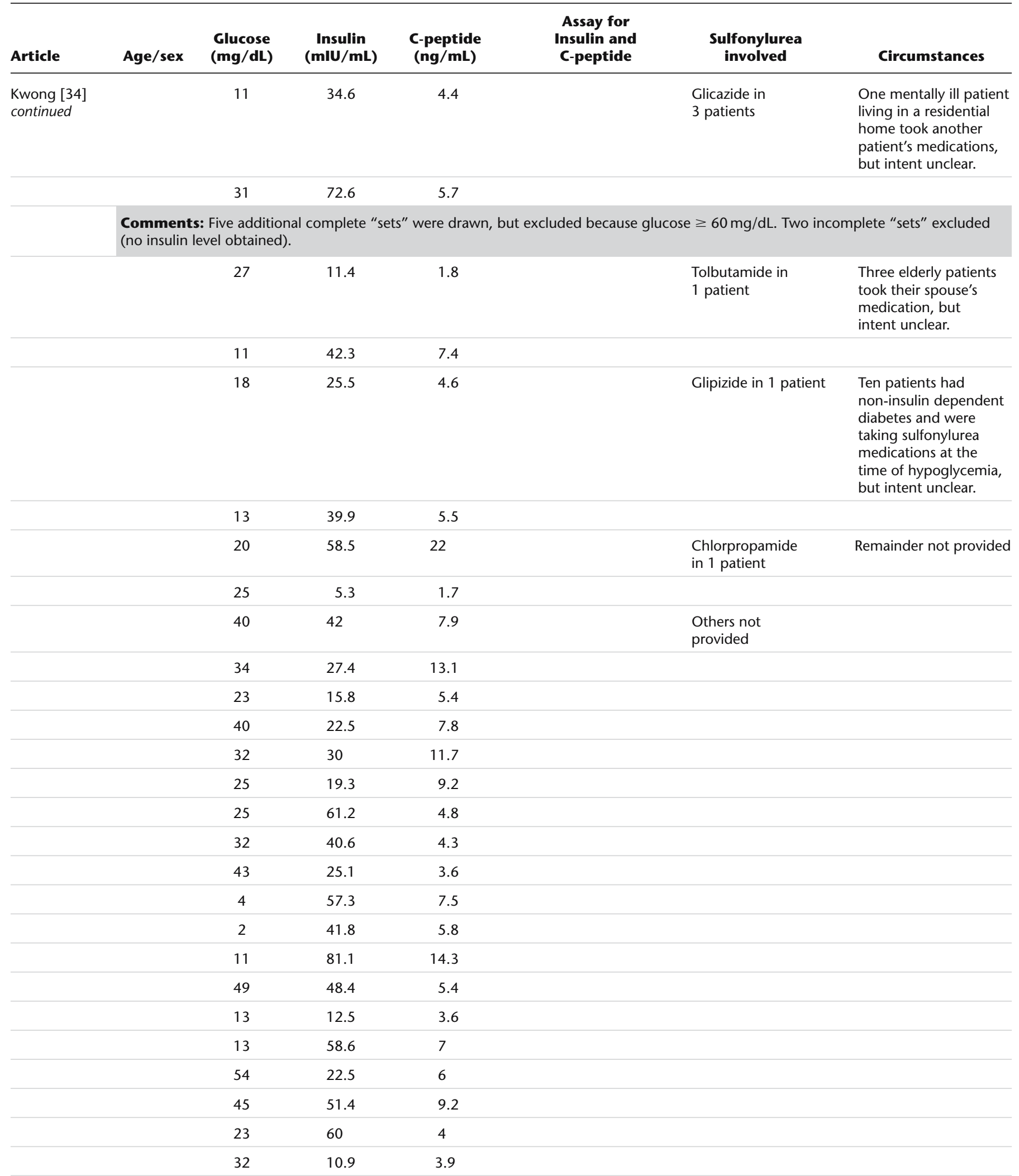




\section{Table 1: (Continued)}

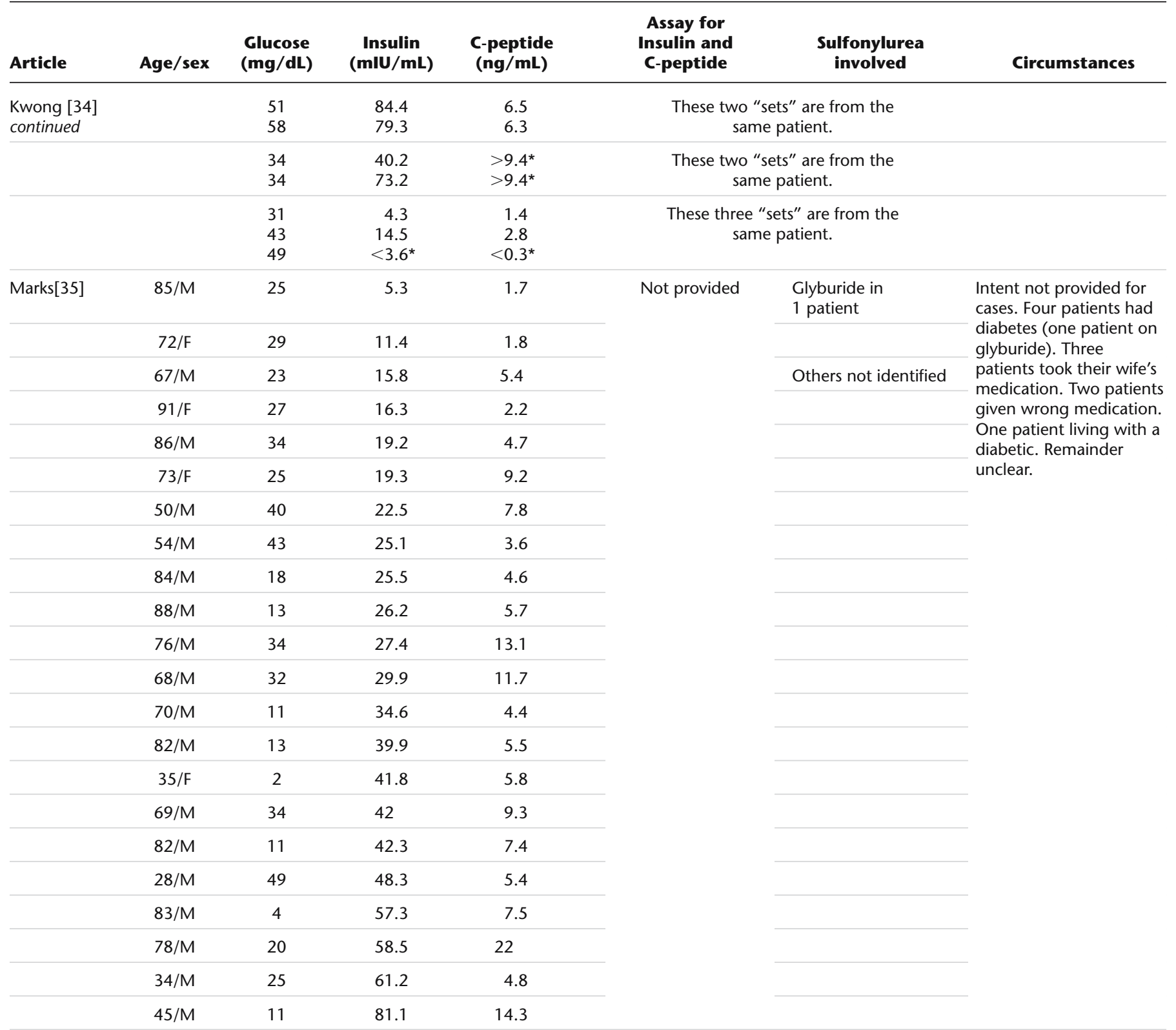

Comments: Unknown if patients received therapy before levels were drawn. Five additional patients listed in article, but 1 was excluded because of no accompanying insulin level, one excluded because of renal failure, and 3 excluded because of glucose $\geq 60 \mathrm{mg} / \mathrm{dL}$.

\begin{tabular}{|c|c|c|c|c|c|c|c|}
\hline McDermott[36] & $67 / F$ & 30 & 22 & 12 & Not provided & Glyburide & $\begin{array}{l}\text { Inadvertently dispensed } \\
\text { instead of metoprolol }\end{array}$ \\
\hline & \multicolumn{7}{|c|}{ Comments: Unknown if patient received therapy before levels were drawn. } \\
\hline \multirow[t]{4}{*}{ Ziegler[37] } & & 34 & 25 & 2.6 & Not provided & Glyburide & $\begin{array}{l}\text { Intentional ingestion } \\
\text { in non-diabetic }\end{array}$ \\
\hline & & 37 & 16 & 1.6 & & & \\
\hline & & 19 & 40 & 2.4 & & & \\
\hline & & 36 & 26 & 2.8 & & & \\
\hline
\end{tabular}


Table 1: (Continued)

\begin{tabular}{|c|c|c|c|c|c|c|c|}
\hline Article & Age/sex & $\begin{array}{c}\text { Glucose } \\
(\mathrm{mg} / \mathrm{dL})\end{array}$ & $\begin{array}{l}\text { Insulin } \\
(\mathbf{m I U} / \mathbf{m L})\end{array}$ & $\begin{array}{c}\text { C-peptide } \\
\text { (ng/mL) }\end{array}$ & $\begin{array}{l}\text { Assay for } \\
\text { Insulin and } \\
\text { C-peptide }\end{array}$ & $\begin{array}{l}\text { Sulfonylurea } \\
\text { involved }\end{array}$ & Circumstances \\
\hline \multirow{3}{*}{$\begin{array}{l}\text { Ziegler[37] } \\
\text { continued }\end{array}$} & & 37 & 29 & 4 & & & \\
\hline & & 43 & 40 & 6.4 & & & \\
\hline & \multicolumn{7}{|c|}{ Comments: Unknown if patient received therapy before levels were drawn. Levels obtained on 6 separate admissions for hypoglycemia } \\
\hline \multicolumn{8}{|c|}{ * Non-exact levels, thus excluded from group comparisons. } \\
\hline \multicolumn{8}{|c|}{ † This C-peptide level was excluded from all calculations. See text. } \\
\hline \multicolumn{8}{|c|}{$\ddagger$ Glucose, insulin, and C-peptide levels reported as means only and excluded from calculations, but included in range descriptions. } \\
\hline
\end{tabular}

Table 2: Descriptive statistics for all articles meeting inclusion criteria

\begin{tabular}{|c|c|c|c|c|}
\hline & $\begin{array}{l}\text { Number of } \\
\text { reported values/ } \\
\text { total patients } \\
\text { described* }\end{array}$ & Range & Median & Mean ( \pm SD) \\
\hline Age (years) & $41 / 76(54 \%)$ & 3 hours to $>91$ & 67 & $59(25)$ \\
\hline \multirow[t]{2}{*}{ Sex } & $\begin{array}{c}41 / 76(54 \%) \\
63 \% \text { male }\end{array}$ & NA & NA & NA \\
\hline & $\begin{array}{l}\text { Number of exact } \\
\text { levels/total } \\
\text { levels reportedt }\end{array}$ & Range & Median & $\begin{array}{c}\text { Mean ( } \pm \text { SD; } \\
95 \% \mathrm{CI})\end{array}$ \\
\hline Glucose (mg/dL) & $94 / 97$ (97\%) & Feb-58 & 29 & $\begin{array}{c}28.6(12.6 ; \\
26.1 \text { to } 31.2)\end{array}$ \\
\hline Insulin ( $\mu \mathrm{IU} / \mathrm{mL})$ & 93/97 (96\%) & $<3.6$ to 1115 & 34 & $\begin{array}{l}54.4(126.3 ; \\
28.3 \text { to } 80.5)\end{array}$ \\
\hline C-peptide (ng/mL) & 90/97 (93\%) & $<0.3$ to $44.1^{\ddagger}$ & $5.7 \ddagger$ & $\begin{array}{c}7.2(6.2 ; \\
5.9 \text { to } 8.5)^{\ddagger}\end{array}$ \\
\hline \multicolumn{5}{|c|}{$\begin{array}{l}\text { † Ninety-seven total "sets" of levels are reported from } 76 \text { patients. Some levels were reported as non-exact levels (ie. reported } \\
\text { as a mean or < or >) and are not included in mean or median calculations. } \\
\ddagger \text { A C-peptide level of } 12,100 \mathrm{ng} / \mathrm{mL} \text { was excluded from all calculations. See text. }\end{array}$} \\
\hline
\end{tabular}

diagnosis of hyperinsulinism in a hypoglycemic setting have not been established [14].

Author opinions and assay methods vary when it comes to suggested insulin and C-peptide cut-off levels needed to diagnose endogenous insulin hypersecretion. And it should be noted that these opinions fail to provide reference to actual values obtained in sulfonylurea-induced hypoglycemia. Service recommends using insulin levels $\geq 6 \mu \mathrm{IU} / \mathrm{mL}$ ( $41.7 \mathrm{pmol} / \mathrm{L})$ using a double-antibody radioimmunoassay (or $\geq 3 \mu \mathrm{IU} / \mathrm{mL}$ (20.8 pmol/L) using a immunochemiluminometric assay and C-peptide levels of $\geq 0.6 \mathrm{ng} / \mathrm{mL}$ $(0.2 \mathrm{nmol} / \mathrm{L})$ in the hypoglycemic setting (blood glucose $\leq 45$ $\mathrm{mg} / \mathrm{dL}$ and patient symptomatic) $[5,10,11]$. Marks and Teale do not provide assay specifics, but Marks and Teale recommend a cut-off of $>5 \mu \mathrm{IU} / \mathrm{mL}(34.7 \mathrm{pmol} / \mathrm{L})$ for insulin, $>0.9 \mathrm{ng} / \mathrm{mL}(0.3 \mathrm{nmol} / \mathrm{L})$ for C-peptide, and blood glucose $<54 \mathrm{mg} / \mathrm{dL}$ [4].
We found a wide range of insulin and C-peptide levels in reported cases of sulfonylurea-induced hypoglycemia. If we apply the extremes of the above thresholds (insulin $\geq 6$ or $\geq 3 \mu \mathrm{IU} / \mathrm{mL}$; C-peptide $\geq 0.9$ or $0.6 \mathrm{ng} / \mathrm{mL}$ ) to the values obtained in our study, 5 insulin levels fall below $6 \mu \mathrm{IU} / \mathrm{mL} ; 1$ insulin level $(<3.6 \mu \mathrm{IU} / \mathrm{mL})$ was possibly below $3 \mu \mathrm{IU} / \mathrm{mL}$; and 1 C-peptide level was below both 0.9 and $0.6 \mathrm{ng} / \mathrm{mL}$. Table 4 shows the details of these cases.

All "sets" with blood glucose $<49 \mathrm{mg} / \mathrm{dL}(2.7 \mathrm{mmol} / \mathrm{L})$ had insulin levels $\geq 3 \mu \mathrm{IU} / \mathrm{mL}$ (lowest $3.9 \mu \mathrm{IU} / \mathrm{mL} ; 1.3 \mathrm{nmol} / \mathrm{L}$ ) and Cpeptide levels $>0.9 \mathrm{ng} / \mathrm{mL}$ and $\geq 0.6 \mathrm{ng} / \mathrm{mL}$ (lowest $1.4 \mathrm{ng} / \mathrm{mL}$; $0.5 \mathrm{nmol} / \mathrm{L})$. Only 4 "sets" with blood glucose $<49 \mathrm{mg} / \mathrm{dL}$ had insulin levels $<6 \mu \mathrm{IU} / \mathrm{mL}$. These 4 "sets" are also shown in Table 4. In "sets" with glucose $\geq 49 \mathrm{mg} / \mathrm{dl}$, insulin was $\geq 22.5 \mu \mathrm{IU} / \mathrm{mL}$ and C-peptide was $\geq 5.4 \mathrm{ng} / \mathrm{mL}$ in all but 1 "set". Table 5 provides details of all "sets" with blood glucose $\geq 49 \mathrm{mg} / \mathrm{dL}$. Because 
Table 3: Comparison between BEFORE therapy, AFTER therapy, and UNKNOWN groups

\begin{tabular}{|c|c|c|c|}
\hline & BEFORE therapy & AFTER therapy & UNKNOWN \\
\hline \multicolumn{4}{|l|}{ Age (years) } \\
\hline mean $( \pm S D ; 95 \% \mathrm{Cl})$ & $49.7 \pm 29.0$ & $52.2 \pm 31.2$ & $65.4 \pm 20.0$ \\
\hline range & $0.5-90$ & $3 \mathrm{hrs}-85$ & $>21->91$ \\
\hline $\begin{array}{l}\text { Number of patients with ages reported/ } \\
\text { number of patients in group }\end{array}$ & $8 / 9^{*}$ & $6 / 7^{*}$ & $26 / 61$ \\
\hline Sex (\% male) & 63 & 50 & 69 \\
\hline $\begin{array}{l}\text { Number of patients with sex reported/ } \\
\text { number of patients in group }\end{array}$ & $8 / 9^{*}$ & $6 / 7^{*}$ & $26 / 61$ \\
\hline \multicolumn{4}{|l|}{ Glucose $(\mathrm{mg} / \mathrm{dL})$} \\
\hline mean $( \pm S D ; 95 \% \mathrm{Cl})$ & $\begin{array}{l}24.3( \pm 7.3 \\
18.7 \text { to } 30.0)\end{array}$ & $\begin{array}{c}33.1( \pm 9.8 \\
28.2 \text { to } 38.0)\end{array}$ & $\begin{array}{l}28.0( \pm 13.5 \\
24.7 \text { to } 31.3)\end{array}$ \\
\hline median & 23 & 30.5 & 29 \\
\hline range & $13 / 36$ & $20 / 52$ & 2 to 58 \\
\hline $\begin{array}{l}\text { Number of exact glucose measurements } \\
\text { provided and used in calculations/ } \\
\text { total number of glucose levels reported } \\
\text { in group }\end{array}$ & 9 of 9 & $18 / 18$ & $67 / 70$ \\
\hline \multicolumn{4}{|l|}{ Insulin ( $\mu$ IU/mL) } \\
\hline mean $( \pm S D ; 95 \% \mathrm{Cl})$ & $\begin{array}{l}36.6( \pm 26.2 ; \\
16.5 \text { to } 56.7)\end{array}$ & $\begin{array}{l}126.7( \pm 278.1 \\
0 \text { to } 265.0)\end{array}$ & $\begin{array}{l}37.1( \pm 21.8 \\
31.7 \text { to } 42.5)\end{array}$ \\
\hline median & 30 & 37 & 32.3 \\
\hline range & $3.9-85.8$ & $14.6-1115$ & $<3.6-102$ \\
\hline $\begin{array}{l}\text { Number of exact insulin measurements } \\
\text { provided and used in calculations / } \\
\text { total number of insulin levels reported } \\
\text { in group }\end{array}$ & 9 of 9 & $18 / 18$ & $66 / 67$ \\
\hline \multicolumn{4}{|l|}{ C-peptide $(\mathrm{ng} / \mathrm{mL})^{\dagger}$} \\
\hline mean $( \pm S D ; 95 \% \mathrm{Cl})$ & $\begin{array}{l}5.4( \pm 4.6 \\
1.5 \text { to } 9.2)\end{array}$ & $\begin{array}{l}10.3( \pm 10.5 \\
5.1 \text { to } 15.4)\end{array}$ & $\begin{array}{l}6.5( \pm 4.3 \\
5.4 \text { to } 7.6)\end{array}$ \\
\hline median & 4.1 & 6.3 & 5.6 \\
\hline range & $1.5-16.2$ & $3.4-44.1$ & $<0.3-22$ \\
\hline $\begin{array}{l}\text { Number of exact C-peptide measurements } \\
\text { provided and used in calculations/ total } \\
\text { number of C-peptide levels reported } \\
\text { in group }\end{array}$ & $8 / 9^{\dagger}$ & $18 / 18$ & $64 / 70$ \\
\hline \multicolumn{4}{|c|}{$\begin{array}{l}\text { * Because the patient in the article by Shima et al. [15] had "sets" of levels included in both the BEFORE and AFTER therapy groups, this patient } \\
\text { was excluded from age and sex calculations. } \\
\dagger \text { A C-peptide level of } 12,100 \mathrm{ng} / \mathrm{mL} \text { was excluded from the BEFORE therapy group. See text. }\end{array}$} \\
\hline
\end{tabular}

hypoglycemic symptoms may begin at glucose levels of $<60$ $\mathrm{mg} / \mathrm{dl}[10]$, clinicians initially faced with a hypoglycemic patient may not have the luxury of confirming that glucose is $<49 \mathrm{mg} / \mathrm{dL}$ before levels are obtained.

Before blood is obtained to measure insulin and C-peptide levels or before the diagnosis of hyperinsulinemia is entertained, patients may receive hypoglycemic therapy. However, even patients in the AFTER therapy group had elevated insulin and C-peptide levels, and they overlapped with insulin and C-peptide levels in the BEFORE therapy group. Most patients in the AFTER therapy group received intravenous glucose, but oral glucose, octreotide, and glucagon were also administered. Because there were only 7 patients in the AFTER therapy group, comparisons between these different hypoglycemic therapies was not possible. Interestingly, the patient in the article by Thoma had the 2 highest insulin levels, and the patient had the second highest C-peptide level of all the groups [30]. This may be related to the administration of $5 \mathrm{mg}$ of intravenous glucagon $[39,40]$. Glucagon stimulates insulin 
Table 4: Details for all "sets" with levels below threshold values of $6 \mu \mathrm{lU} / \mathrm{mL}(41.7 \mathrm{pmol} / \mathrm{L})$ for insulin or $0.9 \mathrm{ng} / \mathrm{mL}(0.3 \mathrm{nmol} / \mathrm{L})$ for C-peptide

\begin{tabular}{|c|c|c|c|c|c|c|}
\hline Article & Age/sex & $\begin{array}{l}\text { Glucose } \\
\text { (mg/dL) }\end{array}$ & $\begin{array}{l}\text { Insulin } \\
(\mu \mathrm{IU} / \mathrm{ml})\end{array}$ & $\begin{array}{l}\text { C-peptide } \\
(\mathbf{n g} / \mathbf{m L})\end{array}$ & $\begin{array}{c}\text { Sulfonylurea } \\
\text { involved }\end{array}$ & Comments \\
\hline \multicolumn{7}{|c|}{ Levels drawn BEFORE hypoglycemic therapy administered } \\
\hline Labib[19] & $90 / \mathrm{F}$ & 13 & 3.9 & 2.5 & Glyburide & \\
\hline \multicolumn{7}{|c|}{ Levels where it is UNKNOWN when levels drawn with respect to hypoglycemic therapy } \\
\hline \multirow[t]{3}{*}{ Kwong[34] } & Unknown & 25 & 5.3 & 1.7 & Unknown & \multirow{4}{*}{$\begin{array}{l}\text { These } 2 \text { "sets" } \\
\text { are from the } \\
\text { same patient }\end{array}$} \\
\hline & & 31 & 4.3 & 1.4 & & \\
\hline & & 49 & $<3.6$ & $<0.3$ & & \\
\hline Marks[35] & $85 / F$ & 25 & 5.3 & 1.7 & Unknown & \\
\hline
\end{tabular}

Table 5: Details for all "sets" with glucose levels $\geq 49 \mathrm{mg} / \mathrm{dl}(2.7 \mathrm{mmol} / \mathrm{l})$

\begin{tabular}{|c|c|c|c|c|c|c|}
\hline Article & Age/sex & $\begin{array}{c}\text { Glucose } \\
(\mathrm{mg} / \mathrm{dL})\end{array}$ & $\begin{array}{l}\text { Insulin } \\
(\mu \mathrm{IU} / \mathbf{m L})\end{array}$ & $\begin{array}{c}\text { C-peptide } \\
\text { (ng/mL) }\end{array}$ & $\begin{array}{c}\text { Sulfonylurea } \\
\text { involved }\end{array}$ & Comments \\
\hline \multicolumn{7}{|c|}{ Levels drawn AFTER hypoglycemic therapy administered } \\
\hline Goudie[27] & $56 / \mathrm{M}$ & 52 & 50 & 11.4 & Glyburide & \\
\hline Shima[15] & $23 / \mathrm{F}$ & 50 & 40 & 7.7 & Tolbutamide & \\
\hline \multicolumn{7}{|c|}{ Levels where it is UNKNOWN when levels drawn with respect to hypoglycemic therapy } \\
\hline \multirow[t]{5}{*}{ Kwong[34] } & Unknown & 49 & 48.4 & 5.4 & Unknown & \multirow{6}{*}{$\begin{array}{l}\text { These } 2 \text { "sets" } \\
\text { are from the } \\
\text { same patient } \\
\text { This "set" is } \\
\text { also in Table } 4\end{array}$} \\
\hline & & 54 & 22.5 & 6 & & \\
\hline & & 51 & 84.4 & 6.5 & & \\
\hline & & 58 & 79.3 & 6.3 & & \\
\hline & & 49 & $<3.6$ & $<0.3$ & & \\
\hline Marks[35] & $28 / \mathrm{M}$ & 49 & 48.3 & 5.4 & Unknown & \\
\hline
\end{tabular}

secretion, and intravenous glucagon administration may exaggerate insulin secretion in patients who are already hypersecreting insulin $[39,42]$. This same dramatic response was not seen in the other patient who received glucagon, and the response, perhaps, is related to a smaller dose or route of administration [27].

Some could argue that measuring insulin and C-peptide levels in hypoglycemia of uncertain cause is unnecessary when sulfonylurea screens are employed as part of the diagnostic work-up. However, not all sulfonylureas are detected by screens, and negative screens do not rule out sulfonylurea-induced hypoglycemia $[21,26,30,36,43]$. Additionally, quinine, non-sulfonylurea diabetic medications such as repalginide, and nateglinide can produce hypoglycemia with elevated insulin and C-peptide levels, but these medications do not appear on sulfonylurea screens [43-45].

As with all article reviews there are limitations to this study. Our search was limited to Medline and articles written in English. The non-English literature may include other medications that would produce different results. However, our review included all sulfonylurea medications available in the US, all but one of those available in Canada (gliclazide), and almost half of those available in other countries [46]. A second limitation includes the values reported in the articles and that insulin and C-peptide levels were measured in several laboratories using different assays. It is possible that there were unreported insulin and $\mathrm{C}$-peptide values that were below that individual laboratory detection limits. A third limitation is the small number of patients available for before and after therapy comparisons. Inclusion of additional levels might alter group comparisons. But given the large overlap between groups, we consider this unlikely. A fourth limitation is that we were limited to the medical details provided in the reports. It is possible some patients had unreported hepatic or renal failure that affected insulin and C-peptide levels. We cannot predict how these unrecognized conditions might alter our results. Finally, all patients did not have confirmatory studies to detect the presence of sulfonylureas. Although unlikely, given the history and laboratory findings, it is 
possible that some of these patients did not have sulfonylureainduced hypoglycemia.

\section{CONCLUSIONS}

There is a lack of information regarding insulin and C-peptide levels observed in sulfonylurea-induced hypoglycemia: so what constitutes "elevated" levels? Based on our study, with the exception of 1 insulin and 1 C-peptide level (both from the same "set" with an associated blood glucose level of $49 \mathrm{mg} / \mathrm{dL}$ ), all insulin levels, based on our study, were $\geq 3.9 \mu \mathrm{IU} / \mathrm{mL}(27.1 \mathrm{pmol} / \mathrm{L})$, and all C-peptide levels were $\geq 1.4 \mathrm{ng} / \mathrm{mL}(0.5 \mathrm{nmol} / \mathrm{L})$. Therefore, serum insulin $\geq 3.9 \mu \mathrm{IU} / \mathrm{mL}$ and C-peptide $\geq 1.4 \mathrm{ng} / \mathrm{mL}$ in a patient with a blood glucose $<49 \mathrm{mg} / \mathrm{dl}$ are consistent with sulfonylurea-induced hypoglycemia. Except for the one aforementioned "set," this relationship also held true for "sets" with blood glucose from $\geq 49$ to $<60 \mathrm{mg} / \mathrm{dl}$.

Glucose, insulin, and C-peptide levels tended to be higher if obtained after hypoglycemic therapy, but there is a large amount of overlap with levels obtained before therapy. Obtaining levels before or after hypoglycemic therapy did not affect interpretation of those levels because all "sets" in the BEFORE and AFTER therapy groups had insulin and C-peptide levels $\geq 3.9 \mu \mathrm{IU} / \mathrm{mL}$ and $\geq 1.4 \mathrm{ng} / \mathrm{mL}$ respectively.

The authors have no potential financial conflicts of interest to report.

\section{REFERENCES}

1. Horwitz, D. L. Factitious and artifactual hypoglycemia. Endocrinol Metab Clin North Am 1989;18(1):203-10.

2. Wallach, J. Laboratory diagnosis of factitious disorders. Arch Intern Med 1994;154(15):1690-1696.

3. Polonsky, K. S. A practical approach to fasting hypoglycemia. N Engl J Med 1992;326(15):1020-1021.

4. Marks, V. and Teale, J. D. Investigation of hypoglycaemia. Clin Endocrinol (Oxf) 1996;44(2):133-136.

5. Service, F. J. Hypoglycemic disorders. N Engl J Med 1995;332(17):1144-1152.

6. Cryer PE. Glucose homeostasis and hypoglycemia. Larsen PR, Kronenberg HM, Melmed S, and Polonsky KS (Eds). Williams Textbook of Endocrinology. 10th edition. Philadelphia, PA: W.B. Saunders, 2003:1585-1618.

7. Wolf LR, Smeeks F, and Policastro M. Oral hypoglycemic agents. Ford MD, Delaney KA, Ling LJ, and Erickson T (Eds). Clinical Toxicology. Philladelphia, PA: W.B. Saunders, 2001:423-432.

8. Bosse GM. Antidiabetic and hypoglycemic agents. Goldfrank LR, Flomenbaum NE, Lewin NA, Howland M, Hoffman RS, and Nelson LS (Eds). Goldfrank's Toxicologic Emergencies. 7th edition. New York, NY: McGraw-Hill, 2002:593-605.

9. Gama, R., Teale, J. D., and Marks, V. Best practice No 173: clinical and laboratory investigation of adult spontaneous hypoglycaemia. J Clin Pathol 2003;56(9):641-646.
10. Service, F. J. Hypoglycemia. Endocrinol Metab Clin North Am 1997;26(4):937-955.

11. Service, F. J. Diagnostic approach to adults with hypoglycemic disorders. Endocrinol Metab Clin North Am 1999;28(3):519-532.

12. Melani, F., Rubenstein, A. H., Oyer, P. E., and Steiner, D. F. Identification of proinsulin and C-peptide in human serum by a specific immunoassay. Proc Natl Acad Sci USA

1970;67(1):148-155.

13. Rendell, M. The expanding clinical use of C-peptide radioimmunoassay. Acta Diabetol Lat 1983;20(2):105-113.

14. Chevenne, D., Trivin, F., and Porquet, D. Insulin assays and reference values. Diabetes Metab 1999;25(6):459-476.

15. Shima K, Tanake R, Nakamura J, Okada Y, and Kumahara Y. A case of factitious hypoglycemia induced by selfadministraion of sulfonylurea. Jap J Med 1978;17(2):138-143.

16. Hussain, K., Mundy, H., Aynsley-Green, A., and Champion, M. A child presenting with disordered consciousness, hallucinations, screaming episodes and abdominal pain. Eur J Pediatr 2002;161(2):127-129.

17. Cryer, P. E. Hypoglycemia of obscure cause. Hosp Pract 1992;27(8):119-122; 125-126; 129-32.

18. Krentz, A. J., Boyle, P. J., Justice, K. M., Wright, A. D., and Schade, D. S. Successful treatment of severe refractory sulfonylurea-induced hypoglycemia with octreotide. Diabetes Care 1993;16(1):184-186.

19. Labib, M. and Marks, V. Inadvertent intake of sulphonylurea. Ann Clin Biochem 1990;27 (Pt 4):382-383.

20. Ludman, P., Mason, P., and Joplin, G. F. Dangerous misuse of sulphonylureas. $\mathrm{Br}$ Med J (Clin Res Ed) 1986;293 (6557):1287-1288.

21. Manning, P. J., Espiner, E. A., Yoon, K., Drury, P. L., Holdaway, I. M., and Bowers, A. An unusual cause of hyperinsulinaemic hypoglycaemia syndrome. Diabet Med 2003;20(9):772-776.

22. Owen, L., Ellis, M., and Shield, J. Deliberate sulphonylurea poisoning mimicking hyperinsulinaemia of infancy. Arch Dis Child 2000;82(5):392-3.

23. Sener, A., Gillet, C., Verhelst, J., DeBoeck, K., Mahler, C., and Malaisse, W. J. Factitious hypoglycaemia documented by a modified assay for the measurement of plasma sulphonylurea. Diabet Med 1995;12(5):433-455.

24. Sledge, E. D. and Broadstone, V. L. Hypoglycemia due to a pharmacy dispensing error. South Med J 1993;86(11): 1272-1273.

25. Christesen, H. B. and Melander, A. Prolonged elimination of tolbutamide in a premature newborn with hyperinsulinaemic hypoglycaemia. Eur J Endocrinol 1998;138(6):698-701.

26. Earle, K. E., Rushakoff, R. J., and Goldfine, I. D. Inadvertent sulfonylurea overdosage and hypoglycemia in an elderly woman: failure of serum hypoglycemia screening. Diabetes Technol Ther 2003;5(3):449-451.

27. Goudie, A. M. and Kaye, J. M. Contaminated medication precipitating hypoglycaemia. Med J Aust 2001; 175(5):256-257. 
28. Shenfield, G. M., Boutagy, J. S., and Webb, C. A screening test for detecting sulfonylureas in plasma. Ther Drug Monit 1990; $12(4): 393-397$.

29. Teale JD, Starkey BJ, Marks V, and et al. The prevalence of factitious hypoglycemia due to sulphonylurea abuse in the UK: a preliminary report. Practical Diabetes

1989;6(4):177-178.

30. Thoma, M. E., Glauser, J., and Genuth, S. Persistent hypoglycemia and hyperinsulinemia: caution in using glucagon. Am J Emerg Med 1996;14(1):99-101.

31. Ahlquist, D. A., Nelson, R. L., and Callaway, C. W. Pseudoinsulinoma syndrome from inadvertent tolazamide ingestion. Ann Intern Med 1980;93(2):281-282.

32. Hampton, S. M., Beyzavi, K., Teale, D., and Marks, V. A direct assay for proinsulin in plasma and its applications in hypoglycaemia. Clin Endocrinol (Oxf)

1988;29(1):9-16.

33. Klonoff, D. C., Barrett, B. J., Nolte, M. S., Cohen, R. M., and Wyderski, R. Hypoglycemia following inadvertent and factitious sulfonylurea overdosages. Diabetes Care

1995;18(4):563-567.

34. Kwong, P. Y. and Teale, J. D. Screening for sulphonylureas in the investigation of hypoglycaemia. J R Soc Med

2002;95(8):381-385.

35. Marks, V. and Teale, J. D. Drug-induced hypoglycemia. Endocrinol Metab Clin North Am 1999;28(3):555-577.

36. McDermott, J. H., Kent, P. D., and Service, F. J. 67-year-old woman with recurrent hypoglycemia. Mayo Clin Proc

2001;76(9):939-42.
37. Ziegler, O., Gross, P., Kolopp, M., Pointel, J. P., and Drouin, P. Factitious hypoglycemia mimicking insulinoma. Diabetes Care 1987;10(3):377-8.

38. Bolli, G. B. and Fanelli, C. G. Physiology of glucose counterregulation to hypoglycemia. Endocrinol Metab Clin North Am 1999;28(3):467-493.

39. Samols, E., Marri, G., and Marks, V. Promotion of insulin secretion by glucagon. Lancet 1965;40:415-416.

40. Oakley, N. W., Harrigan, P., Kissebah, A. H., Kissin, E. A., and Adams, P. W. Factors affecting insulin response to glucagon in man. Metabolism 1972;21(11):1001-1007.

41. Marks, V. and Samols, E. Glucagon test for insulinoma: a chemical study in 25 cases. J Clin Pathol 1968;21(3):346-352.

42. Kumar, D., Mehtalia, S. D., and Miller, L. V. Diagnostic use of glucagon-induced insulin response. Studies in patients with insulinoma or other hypoglycemic conditions. Ann Intern Med 1974;80(6):697-701.

43. Klonoff, D. C. A flaw in the use of sulfonylurea screening to diagnose sulfonylurea overdosages. Diabetes Technol Ther 2003;5(3): $453-454$.

44. Seltzer, H. S. Drug-induced hypoglycemia. A review of 1418 cases. Endocrinol Metab Clin North Am 1989;18(1):163-183.

45. Hirshberg, B., Skarulis, M. C., Pucino, F., Csako, G., Brennan, R., and Gorden, P. Repaglinide-induced factitious hypoglycemia. J Clin Endocrinol Metab 2001;86(2):475-477. 46. MICROMEDEX ${ }^{\circledR}$ Healthcare Series, (electronic version). Thompson Micromedix, Greenwood Village, CO, USA. Available at http://0-www.thompsonhc.com.library.uchsc.edu:80. (Accessed 1 May 2006). 